\title{
Análise dos Atendimentos de Gestantes na Rede de Atenção Básica de Saúde no Município de São Paulo
}

\author{
Mayara Amanda da Silva ${ }^{1}$, Nathália Barbosa Santos ${ }^{2}$, \\ Caroline de Oliveira Costa Souza Rosa ${ }^{2}$, Marcelo Rodrigues dos Santos ${ }^{3}$, \\ Márcia Ito $^{4}$, Alex B. Vieira ${ }^{1}$, Artur Ziviani ${ }^{2}$, Roberto Massi de Oliveira ${ }^{1}$ \\ ${ }^{1}$ Departamento de Ciência da Computação \\ Universidade Federal de Juiz de Fora (UFJF) \\ ${ }^{2}$ Laboratório Nacional de Computação Científica (LNCC) \\ ${ }^{3}$ Universidade Federal de Minas Gerais \\ ${ }^{4}$ Faculdade de Tecnologia de São Paulo (Fatec-SP) \\ mayara.amanda@ice.ufjf.br, nsantos@lncc.br, carolrosa@lncc.br, \\ marrsantos@terra.com.br,m.ito@uol.com.br, alex.vieira@ufjf.edu.br, \\ ziviani@lncc.br, rmassi@ice.ufjf.br
}

\begin{abstract}
Resumo. Neste artigo, são analisadas as trajetórias de atendimento de pacientes na rede de atenção básica de saúde no município de São Paulo. Avaliar como é a trajetória dos pacientes do SUS, seja entre os atendimentos, seja entre as unidades de atendimento, é uma atividade importante para o entendimento e aprimoramento do sistema. Assim, neste artigo, foca-se num contexto de alta relevância social representado pela avaliação da trajetória de gestantes atendidas pelo SUS no município de São Paulo durante os anos de 2014 e 2015. São analisadas as trajetórias dessas gestantes em seus atendimentos e entre as unidades do sistema de saúde. Acredita-se que as análises apresentadas possam auxiliar em um melhor entendimento do sistema e subsidiar decisões para o aprimoramento do sistema de saúde atual.
\end{abstract}

\begin{abstract}
In this paper, we analyze patient trajectories in the primary healthcare network in the city of São Paulo. Assessing the trajectory of SUS patients, be it between appointments or between care units, is an important activity for understanding and improving the system. Thus, in particular, we focus on a context of high social relevance represented by the evaluation of the trajectory of pregnant women attended by SUS in the city of São Paulo during the years 2014 and 2015. We analyze the trajectories of these pregnant women through the system in their care and between the basic health units. We believe that the presented analyses can assist in a better understanding of the system and support decisions for the improvement of the current health system.
\end{abstract}

\section{Introdução}

O sistema público de saúde no Brasil, denominado por Sistema Único de Saúde (SUS), fornece acesso integral, universal e gratuito para toda a população do país [Ministério da Saúde 2019]. O SUS é organizado a partir do conceito de regionalização de atendimentos. Quanto à distribuição espacial de centros de atenção a saúde, existem no Brasil 438 regiões de saúde. Porém, em alguns estados, foram conformadas mesorregiões de saúde a fim de agrupar conjuntos de regiões em busca de oferta de serviços com maior grau de 
complexidade [Saldanha et al. 2019]. Dada essa estrutura baseada em regionalização, conhecer as trajetórias dos pacientes através de seus atendimentos permite entender a oferta de serviços do SUS nas regiões de saúde, sobretudo sob a perspectiva dos pacientes. Além disso, a organização dos serviços apresenta falha de comunicação e de integração entre os níveis de atenção à saúde, problemas os quais as análises da trajetória do paciente podem ajudar a mitigar [Souza et al. 2008].

Avaliar como é a trajetória dos pacientes do SUS, seja entre os procedimentos, seja entre as unidades de atendimento, é uma atividade importante. Porém, mesmo com grande parte dos dados abertos, avaliar o funcionamento de tal sistema complexo não é trivial. Em um país com dimensão continental, como o Brasil, com metrópoles como São Paulo e Rio de Janeiro, a visualização e a compreensão da dinâmica dos atendimentos aos pacientes do SUS passa por uma série de tratamentos de dados, como a subdivisão de registros em casos específicos. Um exemplo de alta relevância do ponto de vista social é a avaliação da trajetória de gestantes atendidas pelo SUS. Nesse caso, pode-se acompanhar a gestante desde a identificação da gravidez, ou sua primeira consulta pré-natal, até a última consulta puerperal. Com esse acompanhamento bem definido, pode-se avaliar a sequência completa de procedimentos e realizar uma comparação com o modelo recomendado pelo SUS, por exemplo. Tal análise de conformidade é importante, pois diversos trabalhos têm indicado que há alta variabilidade de trajetórias reais de cuidados na área de saúde [Kempa-Liehr et al. 2020, Lismont et al. 2016, Baker et al. 2017]. Além disso, essas análises permitem criar subsídios para futuras avaliações e proporcionam ferramentas para melhorar a gestão e alocação dos recursos disponíveis [Pollack et al. 2013].

Assim, neste artigo, avalia-se as trajetórias de gestantes atendidas pelo SUS na cidade de São Paulo durante os anos de 2014 e 2015. Para tal, são adotadas técnicas de ciência de redes e análise de processos. Modelou-se as trajetórias dessas gestantes entre os atendimentos que elas realizam e também a trajetória espacial (entre as unidades de saúde). Por fim, verificou-se como o fluxo teórico, previsto pelo sistema de saúde vigente, e o fluxo prático, derivado das trajetórias das gestantes, se distinguem. E, baseado na literatura pesquisada, a iniciativa de juntar análise sobre os atendimentos e análise espacial num mesmo trabalho, com aplicação no Brasil, é o grande diferencial deste trabalho.

O restante desse trabalho está organizado como segue. A Seção 2 apresenta trabalhos relacionados. Na Seção 3 expõe-se a metodologia usada no estudo deste artigo. Na Seção 4, são apresentados uma caracterização geral dos dados seguida das análises de trajetórias entre os atendimentos realizados e também a trajetória espacial das gestantes. Por fim, na Seção 5, encontram-se as conclusões e a discussão de trabalhos futuros.

\section{Trabalhos Relacionados}

Atualmente, diferentes métodos de análise espacial na saúde coletiva vêm sendo desenvolvidos para detecção de aglomerados espaciais ou espaço-temporais e aplicados ao planejamento e avaliação de uso de serviços de saúde [Bailey 2001].E, tratando-se de melhorias na assistência médica, [Thorwarth and Arisha 2012] afirma que as simulações vem ganhando aceitação dentro dessa área. A proposta do autor foi modelar fluxos de processos de trabalho dos funcionários e de encaminhamento dos pacientes ou eventos no departamento de emergência da Irlanda a fim de investigar o sistema de funcionamento desse estabelecimento, sendo possível determinar eventuais melhorias. Já [Espinoza et al. 
2014] propõem o uso de simulações em tempo real para analisar as operações que são realizadas diariamente nas salas de emergência. Essa proposta visa estudar o impacto do uso de simulações que preveem o desempenho da sala de emergência com uma quantidade limitada de informações, ou seja, o quanto isso influencia na tomada de decisões.

No trabalho de [Ito et al. 2017], utiliza-se um conjunto de dados de procedimentos médicos realizados em todo o Brasil no ano de 2014, para a construção de uma rede de conexões entre médicos e pacientes. A rede construída é analisada a fim de verificar a importância relativa dos médicos na rede e a possibilidade de colaborações entre eles no tratamento de pacientes. Os autores concluem que a utilização das métricas propostas no trabalho foram promissoras para definir prováveis equipes de atendimento, encontrando subconjuntos de médicos com um alto índice de pacientes comuns.

Neste artigo, em contraste, são analisadas as trajetórias de pacientes através de seus atendimentos, com foco particular nas trajetórias de gestantes como estudo de caso.

\section{Preparação da Base de Dados}

A Figura 1 apresenta um resumo das etapas componentes do método adotado neste artigo para a análise de trajetórias das gestantes. A primeira etapa consiste na preparação dos dados, incluindo seu processamento e limpeza, o que será objeto do restante desta seção. Na Seção 4 são apresentadas as duas outras etapas, caracterização de atendimentos e a trajetória das gestantes, tanto por atendimento, quanto por unidades de saúde.

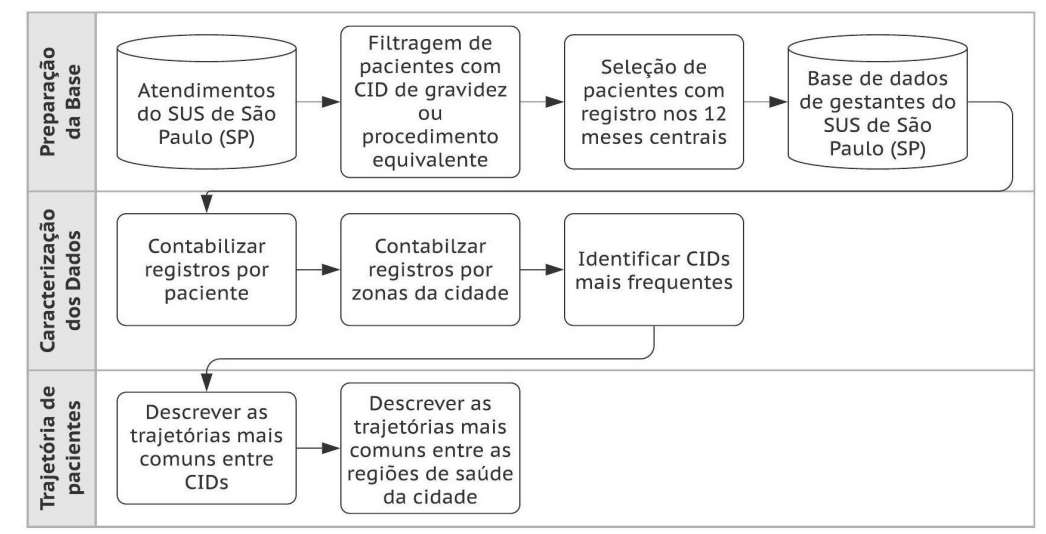

Figura 1. Diagrama das etapas metodológicas adotadas.

Os dados usados neste trabalho são registros de atendimentos de uma base de dados do SUS da cidade de São Paulo (SP), compreendidos entre os meses de janeiro de 2014 e dezembro de 2015. ${ }^{1}$ Esses registros estão divididos em atendimentos com procedimentos, usando os códigos do Sistema de Gerenciamento da Tabela de Procedimentos, Medicamentos e OPM do SUS (SIGTAP) e atendimentos com diagnósticos, usando os identificadores de Classificação Estatística Internacional de Doenças e Problemas Relacionados com a Saúde (CID). Para avaliar a trajetória de um paciente no sistema de saúde, optou-se por analisar dados referentes a gestantes uma vez que se pode avaliar a sequência completa de procedimentos e realizar uma comparação com o modelo recomendado pelo SUS, além de ser um estudo de caso de alta relevância do ponto de vista social.

\footnotetext{
${ }^{1}$ Utilização dos dados aprovada pelo Comitê de Ética e Pesquisa (CAAE: 51038515.6.3001.0086).
} 
Neste trabalho, foram utilizados os registros dos atendimentos com diagnóstico registrado por um CID. Nessa base, há um total de 58.794.547 registros, com os mais variados CID. Foram selecionados 77 CID atribuídos exclusivamente à gestação. Entre todos os registros, 127.846 são relacionados a algum desses 77 CID. Por fim, associadas a esses registros, há 43.724 gestantes com identificadores de pacientes distintos. Destacase que o número de nascidos vivos no município de São Paulo, ${ }^{2}$ segundo o Sistema de Informações de Nascidos Vivos (SINASC) do SUS, foi de 198.125 em 2014 e 198.472 em 2015. Assim, considera-se que a amostra de gestantes usada neste trabalho é expressiva.

A fim de obter somente registros de gestantes com gestação completa durante o período disponível, optou-se por uma filtragem de tempo, como mostrado na Figura 2. Nesse método, os 24 meses da base de dados foram separados em 3 partes: os 6 meses iniciais (de jan/2014 a jun/2014); os 12 meses centrais (de jul/2014 a jun/2015); e os últimos 6 meses (de jul/2015 a dez/2015). Assim, se uma gestante teve atendimento nos 6 primeiros meses, mas não nos 12 meses centrais, então provavelmente a sua gestação se iniciou em 2013 e terminou no início de 2014, de modo que esta paciente não é interessante para este estudo. De forma semelhante, se a paciente teve atendimento nos últimos 6 meses, mas não nos 12 meses centrais, então provavelmente sua gestação se iniciou no fim de 2015 e terminou em 2016, o que também não é proveitoso para esta pesquisa.

Por outro lado, casos como os ilustrados por "Gestação 1", "Gestação 2" e "Gestação 3" na Figura 2 são alvo deste estudo. A "Gestação 1" corresponde aos atendimentos de gestantes cuja gestação iniciou nos 6 primeiros meses e terminaram na parte central da base. Já "Gestação 2" representa os registros de gestantes que começaram sua gestação nos meses centrais da base e cuja gestação terminou nos 6 últimos meses. Por sua vez, "Gestação 3" contempla todas as gestações cujos registros estão na parte central da base, ou seja, entre os meses de julho de 2014 e junho de 2015.

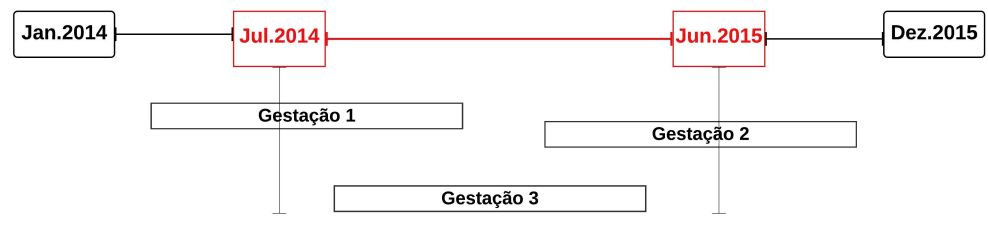

Figura 2. Filtragem temporal para seleção de gestantes da base (2014-2015).

Após identificar as gestantes com ao menos um atendimento durante os 12 meses centrais, foram selecionados os registros de atendimento com diagnóstico referentes a elas, criando a base de dados de gestantes com gestação completa. Foram agregados à essa base os registros de dois procedimentos da tabela SIGTAP ligados a essas mesmas gestantes, que coincidem com um dos CIDs de gestação. Esses procedimentos são o Teste Rápido de Gravidez e o Teste Rápido Para Detecção de HIV em Gestante. Esses dois procedimentos foram escolhidos, pois têm ligação direta ao CID "Z32" (Exame ou Teste de Gravidez). Com isso, tem-se a base final que foi usada neste estudo, referente a 24.916 gestantes.

\footnotetext{
${ }^{2}$ O IBGE apresenta valores diferentes: 190.338 em 2014 e de 191.749 em 2015.
} 


\section{Análise das Trajetórias de Atendimentos das Gestantes}

Nesta seção, apresenta-se a caracterização dos atendimentos às gestantes no período de interesse e a análise das trajetórias das gestantes por esses atendimentos. Nesse contexto, atendimento engloba tanto consultas médicas quanto procedimentos de exames simples, como testes de gravidez ou de detecção de HIV.

\subsection{Caracterização de atendimentos}

Inicialmente, contabilizou-se o número de atendimentos para cada uma das gestantes. A Figura 3(a) apresenta a função de distribuição acumulada (CDF) do número de atendimentos por gestante. Já a Figura 3(b) representa a distribuição de atendimentos realizados por gestante. De acordo com os resultados e por se tratar de um caso discreto, a distribuição geométrica se mostrou mais eficiente, por comportar melhor os dados amostrais. Sendo assim, a Figura 3(b) tem características dessa distribuição e, visualmente inspecionando os resultados, percebe-se que as amostras se comportam conforme a distribuição geométrica de equação $P(X=n)=(1-p)^{n-1} p$, onde $p \approx 0,8$.

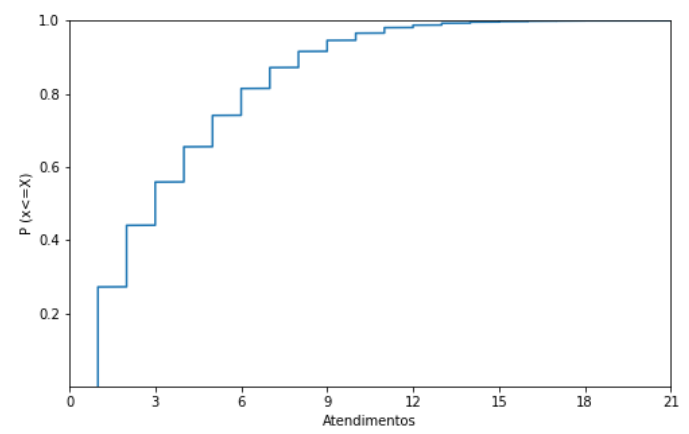

(a) CDF dos atendimentos

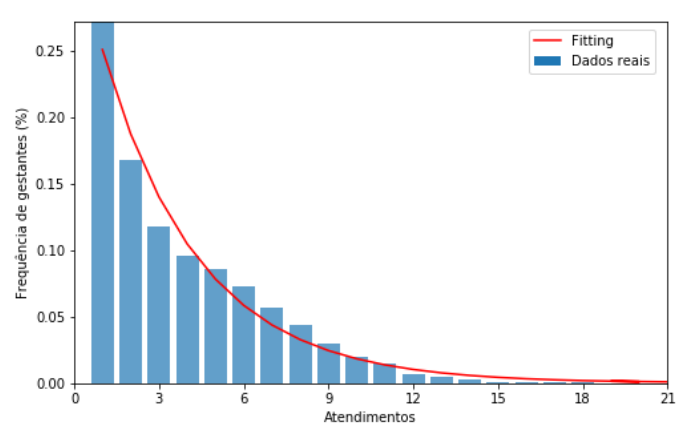

(b) Distribuição geométrica dos atendimentos

Figura 3. Distribuições dos atendimentos por gestante, onde apresentase (a) uma função de distribuição cumulativa (CDF) e (b) uma distribuição geométrica com fitting dos dados sobre o histograma de dados reais.

De forma geral, com base nos resultados da Figura 3, as gestantes avaliadas tiveram um baixo número de atendimentos junto ao sistema de saúde. Ressalta-se que 8 atendimentos é o número indicado pelo Manual Técnico do Pré-natal e Puerpério, proposto pela Secretaria de Estado da Saúde de São Paulo (SES-SP) [São Paulo 2010]. Observa-se que $20 \%$ das gestantes identificadas fizeram pelos menos 6 atendimentos. Nesse conjunto de dados, cerca de $27 \%$ das gestantes fizeram apenas um atendimento e, na média, foram realizados 3.87 atendimentos por gestante, sendo 30 o número máximo de atendimentos feitos por uma gestante.

Alguns dos CIDs registrados na base contêm apenas a categoria do diagnóstico (CID de três dígitos), enquanto outros contêm a subcategoria (quarto dígito). A fim de comparar a frequência dos CIDs, optou-se por considerar somente a categoria dos registros. Por exemplo, se um atendimento continha o CID Z349 ("Supervisão De Gravidez Normal, Não Especificada"), neste primeiro momento considerou-se apenas que ele está ligado à categoria de mais alto nível Z34 (“Supervisão de gravidez normal”).

A Tabela 1 apresenta a distribuição dos diagnósticos por categoria de CID mais comuns no conjunto de dados. Essa tabela mostra os cinco diagnósticos mais comuns, 
uma vez que eles já são responsáveis por aproximadamente $96 \%$ dos atendimentos encontrados no conjunto de dados.

Tabela 1. Diagnósticos mais frequentes por categoria de CID.

\begin{tabular}{|c|c|c|}
\hline Descrição do CID & Frequência absoluta & Frequência relativa \\
\hline \hline Supervisão de gravidez normal & 66.904 & $69,31 \%$ \\
\hline Supervisão de gravidez de alto risco & 13.315 & $13,79 \%$ \\
\hline Exame ou teste de gravidez & 10.783 & $11,17 \%$ \\
\hline Gravidez como achado casual & 1.142 & $1,18 \%$ \\
\hline Gravidez ectópica & 718 & $0,74 \%$ \\
\hline
\end{tabular}

Observa-se na Tabela 1 que os CIDs de supervisão de gravidez representam a maior parte dos diagnósticos, sendo que a supervisão de gravidez normal está relacionada a mais de dois terços dos atendimentos. O número de registros de supervisão de gestação de alto risco também é notável, mas deve-se destacar que a porcentagem de atendimentos não é necessariamente proporcional à porcentagem de pacientes com gestação de alto risco. Isso se dá por dois motivos: Por um lado, espera-se que o número de atendimentos por gestante seja maior nesta classe dada a necessidade de acompanhamento mais frequente da gestação; por outro lado, este não é o único CID voltado para gestações de risco (o quinto CID mais comum, "Gravidez Ectópica" é um exemplo disso). O terceiro diagnóstico mais comum relacionado é, na verdade, o procedimento de teste de gravidez. Diferentemente dos CIDs de supervisão de gravidez, espera-se que cada paciente realize no máximo um teste de gravidez e, por isso, já era esperada uma participação menor deste CID. Percebe-se, no entanto, que a frequência absoluta do teste de gravidez é inferior à metade do número de gestantes. Uma hipótese que justificaria este cenário é a de que o teste de gravidez, por ser um procedimento bem simples e de baixo custo, pode ter sido realizado pela própria gestante, sendo subestimado nessa análise.

Dada a predominância de atendimentos com as duas categorias de CIDs de supervisão de gravidez (normal e de alto risco), buscou-se identificar na base as subcategorias mais comuns em cada caso. Para a categoria relacionada à gravidez normal, tem-se quatro CIDs, distribuídos como apresentado na Figura 4. Observa-se que em mais de $80 \%$ dos casos não há mais detalhes sobre a gestação. Algo semelhante ocorre com a categoria de supervisão de gestação de alto risco, em que 90,7\% dos registros não possuem o identificador de subcategoria e $6.0 \%$ constam como supervisão não especificada.
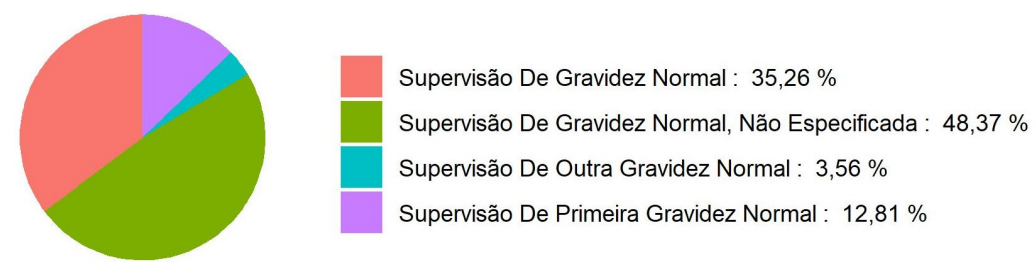

Figura 4. CIDs da categoria "Supervisão de Gravidez Normal” presentes na base.

A Tabela 2 apresenta os diagnósticos mais comuns iniciando ou finalizando o conjunto de dados avaliado, i.e., mais frequentes como primeiro ou último registro de uma paciente, respectivamente. Observa-se que o diagnóstico "Supervisão de Gravidez Normal" é o mais comum, tanto como primeiro quanto como último registro. O "Exame ou teste de gravidez" é o segundo procedimento mais comum iniciando o registro de uma 
gestante. Como explicado anteriormente, é um resultado coerente, pois possivelmente muitas gestantes iniciam o acompanhamento pré-natal a partir de um resultado positivo do teste realizado particularmente. Em $6.39 \%$ das vezes, o teste de gravidez foi o último registro e uma hipótese que justifica isso é a de que essas pacientes podem ter recebido um resultado negativo para o teste de gravidez, encerrando assim o seu acompanhamento. A "Supervisão de gravidez de alto risco" é a terceira categoria mais comum como primeiro registro, mas passa a ser a segunda mais frequente como categoria final da trajetória da paciente, incrementando a participação de $8,51 \%$ para $13,69 \%$. Isso se justifica não só pela participação menor do teste de gravidez como último registro, mas também pelo fato de que muitas vezes a gestação se inicia sem problemas, mas uma condição de risco se desenvolve ao longo das semanas.

Tabela 2. Diagnósticos mais frequentes como primeiro e último registro.

\begin{tabular}{|c|c|c|c|}
\hline $\begin{array}{c}\text { Categorias de CID mais frequentes como } \\
\text { primeiro registro }\end{array}$ & $\begin{array}{c}\text { Freq. } \\
\text { Relativa }\end{array}$ & $\begin{array}{c}\text { Categorias de CID mais frequentes como } \\
\text { último registro }\end{array}$ & $\begin{array}{c}\text { Freq. } \\
\text { Relativa }\end{array}$ \\
\hline \hline Supervisão de gravidez normal & $55,73 \%$ & Supervisão de gravidez normal & $70,66 \%$ \\
\hline Exame ou teste de gravidez & $27,18 \%$ & Supervisão de gravidez de alto risco & $13,69 \%$ \\
\hline Supervisão de gravidez de alto risco & $8,51 \%$ & Exame ou teste de gravidez & $6,39 \%$ \\
\hline Gravidez ectópica & $1,84 \%$ & Gravidez ectópica & $1,83 \%$ \\
\hline Gravidez como achado casual & $1,49 \%$ & Gravidez como achado casual & $1,81 \%$ \\
\hline
\end{tabular}

Considerando os procedimentos de "Exame ou teste de gravidez", "Supervisão de gravidez normal" e "Supervisão de gravidez de alto risco", é possível estabelecer ainda quais foram as especialidades médicas que mais frequentemente realizaram esses registros de atendimento. A Tabela 3 apresenta essas informações ${ }^{3}$. Como poderia se esperar, por ser um procedimento simples, os exames ou testes de gravidez são realizados em sua maioria por um auxiliar de enfermagem. Em outro extremo, a supervisão de uma gravidez de alto risco é acompanhada por um médico especialista em fração significativamente superior à supervisão de uma gravidez considerada normal.

Tabela 3. Especialidades que mais registraram os respectivos CIDs.

\begin{tabular}{|l|l|l|l|l|l|}
\hline \multicolumn{1}{|c|}{ CID Z32 } & Freq. & \multicolumn{1}{|c|}{ CID Z34 } & \multicolumn{1}{c|}{ Freq. } & \multicolumn{1}{c|}{ CID Z35 } & \multicolumn{1}{c|}{ Freq. } \\
\hline Aux. de enfermagem & $41,39 \%$ & Ginecologista e Obstetra & $72,43 \%$ & Ginecologista e Obstetra & $89,68 \%$ \\
\hline Aux. de enfermagem da ESF & $28,40 \%$ & Médico da ESF & $15,81 \%$ & Médico da ESF & $3,46 \%$ \\
\hline Enfermeiro & $12,09 \%$ & Radiologia e DI & $9,78 \%$ & Radiologia e DI & $3,13 \%$ \\
\hline Ginecologista e Obstetra & $7,92 \%$ & Médico Clínico & $0,90 \%$ & Nutricionista & $1,51 \%$ \\
\hline
\end{tabular}

\subsection{Trajetória de atendimentos}

De acordo com Programa de Humanização no Pré-natal e Nascimento, disponibilizado pelo Ministério da Saúde [Ministério da Saúde 2000], o fluxo teórico ideal que deve ser seguido por uma gestante apresenta seis atendimentos pré-natal seguido por um atendimento pós-parto, como ilustra a Figura 5.

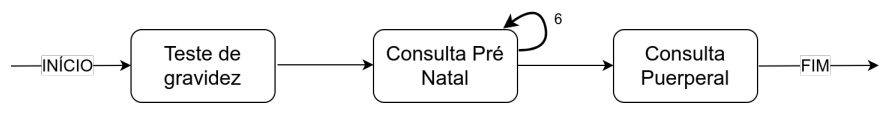

Figura 5. Fluxo teórico de atendimento à gestante recomendado pelo SUS.

${ }^{3}$ ESF: Estratégia e Saúde da Família, DI: Diagnóstico e Imagem 
Para uma análise visual da trajetória de atendimentos foi utilizada a representação através de um mapa de processos. Um mapa de processos é um grafo ponderado e direcionado cujos nós são as atividades existentes e as arestas são criadas quando um mesmo caso realiza uma atividade (nó de origem da aresta) e, em seu próximo registro, realiza outra (nó de destino da aresta). Quanto mais casos repetirem a mesma aresta, maior será o peso dessa aresta. Por exemplo, quando uma gestante realiza um procedimento " $\mathrm{x}$ " no tempo $t$, é criado um nó no mapa de processos. Quando essa gestante realiza um procedimento "y" em $t+1$, também é criado um nó "y" no mapa. Como "y" logicamente sucede "x", é criada uma aresta direcionada " $x$ " $\rightarrow$ " $y$ ". A informação temporal dessa aresta é capturada pelo mapa de processos.

Assim, para as gestantes com mais de um atendimento, foi criado um mapa de processos a partir dos dados do conjunto de dados do período completo (2014-2015). A Figura 6 apresenta uma visualização da sequência de atendimentos a cada gestante identificada na base. Cada linha nessa figura representa a trajetória (sequência) de atendimentos de uma gestante. A primeira gestante encontrada no conjunto de dados é a linha apresentada mais acima, no eixo Y do gráfico. Os círculos apresentados em cada linha representam os atendimentos realizados pela gestante na data correspondente à sua coordenada. Esses círculos estão coloridos de acordo com o código CID do procedimento correspondente. Os 16 tipos de atendimentos apresentados na Figura 6 são responsáveis por $98 \%$ das atividades das gestantes na base de dados avaliada.

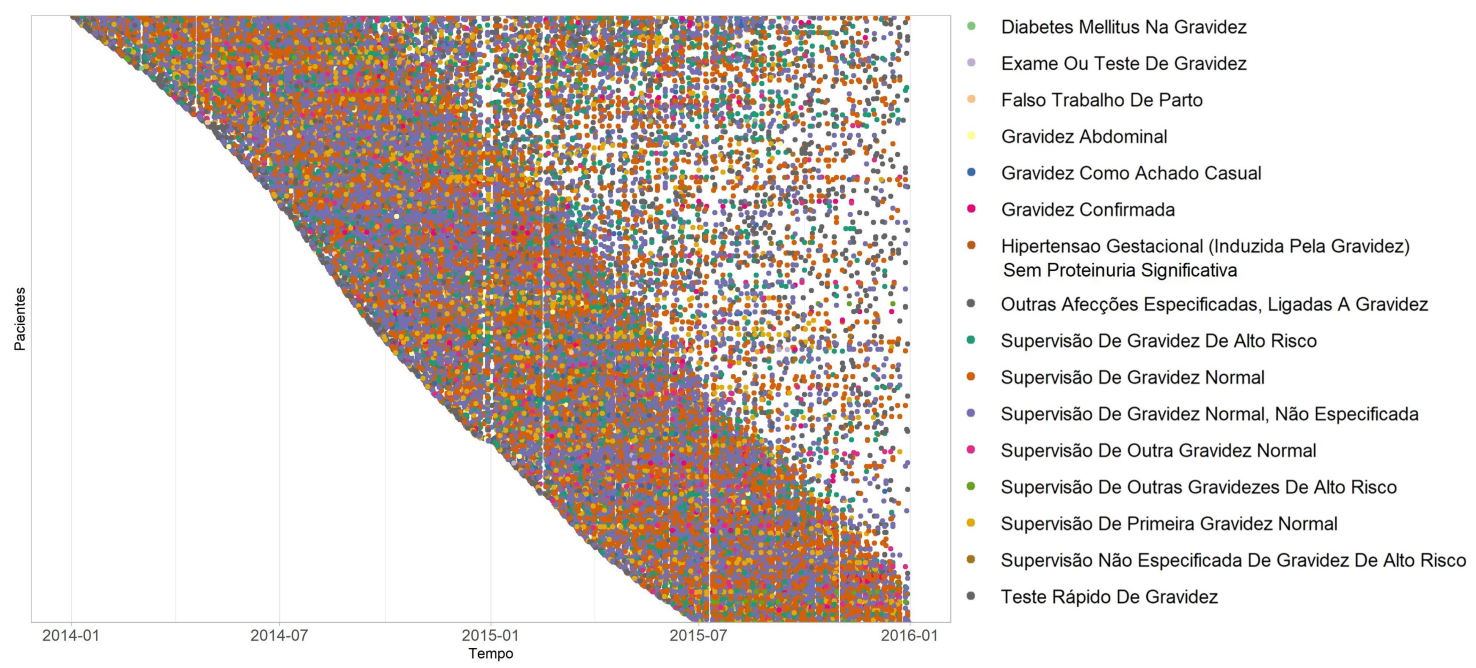

Figura 6. Dotted chart temporal dos atendimentos a gestantes. No eixo horizontal tem-se uma linha do tempo e cada "linha" do eixo vertical representa a sequência de atendimentos a uma gestante, sendo coloridos de acordo com o CID correspondente. Os dados foram filtrados para conter apenas gestantes com ao menos dois atendimentos e inclui $98 \%$ das atividades mais frequentes.

Note que, visualmente, o conjunto de pontos (atendimentos) na Figura 6 tem um adensamento de atividades em um período de aproximadamente nove meses. Porém, em mais detalhes, nota-se que o tempo médio entre o primeiro e o último evento de uma gestante é de 23,5 semanas, com desvio-padrão de 16,33 semanas. Em outras palavras, pelos conjunto de dados analisado, o acompanhamento de uma gestante também é inferior ao desejado, assim como o número médio de atendimentos. 
A Figura 7 apresenta as sequências mais comuns de atendimentos, encontradas entre as 4.507 sequências de atividades diferentes para todas as 24.916 gestantes. Metade dessas sequências mais comuns têm apenas uma atividade. Inclusive, a sequência mais comum entre todas têm apenas um registro, o de "Supervisão de Gravidez Normal, Não Especificada". Assim, essa figura corrobora com os dados apresentados anteriormente na Figura 4. Como apontado anteriormente, também, "Supervisão de Gravidez Normal, Não Especificada" é um dos atendimentos mais comuns.

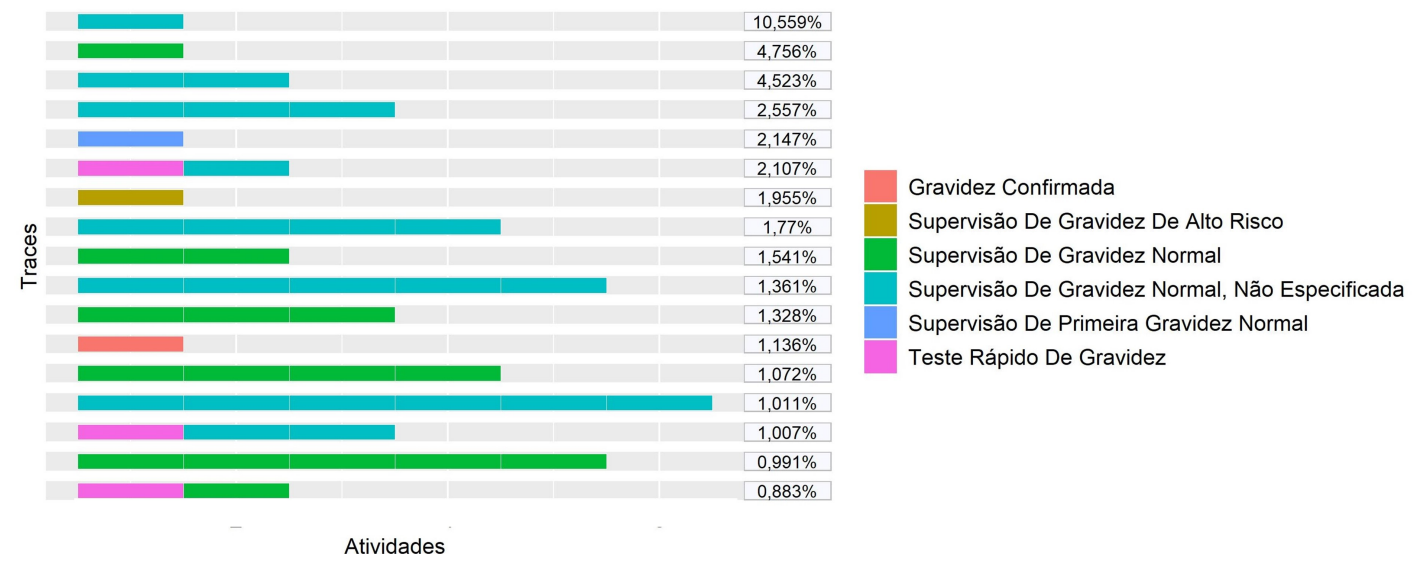

Figura 7. Trajetórias de atendimentos mais frequentes pelas gestantes.

A Figura 8 apresenta o fluxo de atendimento geral para as gestantes entre as categorias de CIDs associadas aos atendimentos do sistema de saúde. Para tornar a figura de mais fácil visualização, foram eliminados os registros de gestantes com apenas um atendimento e selecionou-se apenas os CIDs mais frequentes e responsáveis por $95 \%$ dos registros. A porcentagem nas arestas corresponde à parcela de registros que saem do vértice de origem e o número nos vértices corresponde ao número de atendimentos com aquele CID. Deste modo, tem-se a probabilidade do próximo estado da gestante. Por exemplo, 49,7\% das gestantes que realizaram "Exame ou teste de gravidez" tem como próximo registro "Supervisão de gravidez normal".

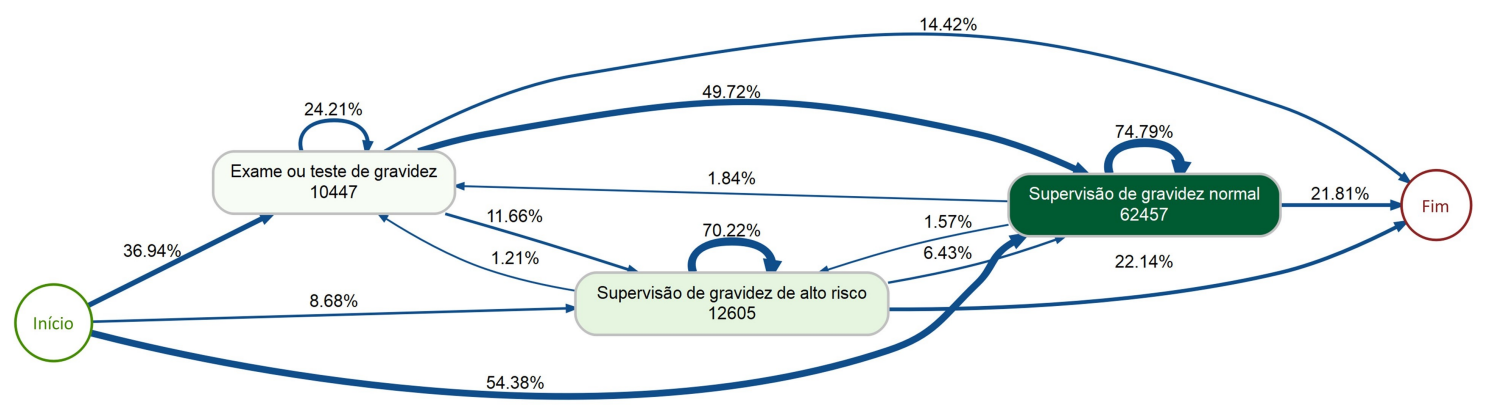

Figura 8. Trajetórias das gestantes pelas categorias de CIDs. O grafo foi gerado considerando apenas gestantes com ao menos dois atendimentos e atividades responsáveis por $95 \%$ dos atendimentos. O número de atendimentos com cada CID é apresentado no respectivo vértice. Os vértices "Início" e "Fim" são virtuais para representar o início e fim de uma sequencia de CIDs, respectivamente. Dado um par de vértices $i \mathbf{e} j$, cada aresta direcionada indica a fração de atendimentos do vértice $i$ que tiveram como atendimento subsequente o vértice $j$. 


\subsection{Trajetória Espacial das Gestantes}

No município de São Paulo, que contava com uma população de 11,25 milhões de habitantes em $2010^{4}$, atuam cinco Coordenadorias de Regiões de Saúde (CRS) ${ }^{5}$ : Centro-Oeste, Leste, Norte, Sudeste e Sul. Essas coordenadorias são responsáveis, além pela coordenação, articulação, organização do sistema de saúde loco-regional e compatibilização dos planos, programas e projetos dos Departamentos Regionais de Saúde (DRS) em função das políticas e diretrizes da SES/SP e dos recursos disponíveis [São Paulo 2020]. A distribuição de renda entre essas regiões não é igualitária, como pode-se observar na Tabela 4 . Nessa tabela, apresenta-se o rendimento médio mensal de uma pessoa, segundo a CRS do Município de São Paulo em $2010^{6}$, e com isso, é possível ver que a região Centro-Oeste é a que apresenta maior renda média, cujo valor é quase quatro vezes maior do que o referente à região Leste, a de menor renda média.

\begin{tabular}{|l|l|l|l|l|l|}
\cline { 2 - 6 } \multicolumn{1}{c|}{} & CRS Centro-Oeste & CRS Sudeste & CRS Sul & CRS Norte & CRS Leste \\
\hline Rendimento & $\mathrm{R} \$ 3.846,24$ & $\mathrm{R} \$ 2.323,73$ & $\mathrm{R} \$ 1.568,30$ & $\mathrm{R} \$ 1.550,09$ & $\mathrm{R} \$ 1.054,47$ \\
\hline
\end{tabular}

Tabela 4. Rendimento nominal médio mensal de pessoas com 10 anos ou mais de idade segundo a CRS do Município de São Paulo em 2010.

A Tabela 5 apresenta o número de gestantes com um único atendimento e o número total de gestantes, para cada uma das regiões de São Paulo. A região CentroOeste é a que tem o maior percentual de gestantes com um único atendimento, com praticamente $47 \%$ delas nessa situação. Nas demais regiões, esse número é igual ou inferior a 26\%. Não se pode afirmar com certeza, mas, conjectura-se que na região Centro-Oeste, por ter uma média de renda mais elevada, como apresentado anteriormente, as gestantes procuram um serviço médico suplementar ao SUS na rede privada, o que justifica o grande número de gestantes com baixo número de atendimentos nessa região.

Tabela 5. Região dos atendimentos de gestantes com uma consulta.

\begin{tabular}{|c|c|c|c|}
\hline Regiões & $\begin{array}{c}\text { Gestantes com apenas } \\
\text { um registro }\end{array}$ & Total de gestantes & Porcentagem \\
\hline \hline Sul & 3.330 & 12.839 & $25,93 \%$ \\
\hline Centro Oeste & 1.672 & 3.566 & $46,88 \%$ \\
\hline Sudeste & 696 & 2.889 & $24,09 \%$ \\
\hline Norte & 565 & 3.238 & $17,44 \%$ \\
\hline Leste & 516 & 2.384 & $21,64 \%$ \\
\hline
\end{tabular}

Também foram avaliadas as trajetórias das gestantes entre as unidades de atendimento do sistema de saúde. Sabe-se que durante o período gestacional, a gestante pode ser atendida em diversas unidades de atendimento. A Figura 9 mostra uma matriz de precedência das regiões dos atendimentos. Neste trabalho, focou-se nas regiões de saúde em que o município de São Paulo é dividido, mas tal abordagem pode ser expandida para traçar a trajetória entre as unidades de atendimento do sistema de saúde. Ao analisar a Figura 9, vê-se que a probabilidade do próximo atendimento ser na mesma região é bastante

\footnotetext{
${ }^{4}$ https://cidades.ibge.gov.br/brasil/sp/sao-paulo/panorama

${ }^{5}$ Atualmente, as CRS Centro e Oeste aparecem separadamente no site da prefeitura (https://www.prefei tura.sp.gov.br/cidade/secretarias/saude/organizacao/index.php? $\mathrm{p}=228575$ ), contabilizando seis zonas. No período do estudo, no entanto, os registros referem-se apenas às cinco citadas no texto.

${ }^{6} \mathrm{De}$ acordo com: https://www.prefeitura.sp.gov.br/cidade/secretarias/saude/epidemiologia_e_informaca o/geoprocessamento_e_informacoes_socioambientais/index.php?p=265358
} 
alta para todas as zonas. Destaca-se que, neste caso, não se considera a probabilidade de um atendimento ser o primeiro ou o último. Por exemplo, dado que uma gestante foi atendida na região Sul e que ela fará um novo atendimento, a probabilidade de que esse atendimento seja feito também na região Sul é de 99,84\%.

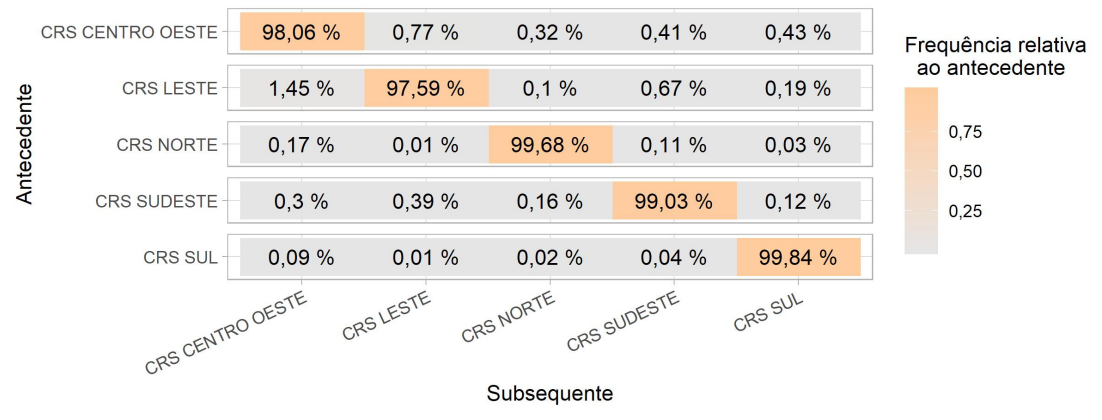

Figura 9. Matriz de precedência das regiões dos atendimentos.

\section{Conclusões e Trabalhos Futuros}

Neste artigo, foram avaliadas as trajetórias dos atendimentos de gestantes atendidas pelo SUS da cidade de São Paulo durante 2014 e 2015. As principais conclusões foram que: (i) menos de $20 \%$ das gestantes realizaram ao menos seis atendimentos; (ii) o diagnóstico "Supervisão de Gravidez Normal" é o mais atribuído aos atendimentos, representando quase $70 \%$ dos registros; (iii) há grande variedade de trajetórias de atendimento; (iv) a região Sul concentra a maior parte das gestantes, enquanto que a região Centro-Oeste tem a maior porcentagem de gestantes com apenas um atendimento; (v) não se observou mobilidade elevada das pacientes entre as regiões de saúde de São Paulo. Pode-se afirmar que as limitações da pesquisa se deram devido ao intervalo restrito em que os dados foram obtidos e pelo fato de só terem sido selecionadas gestantes vinculadas aos registros de atendimentos com CIDs, o que limita muito a quantidade de registros.

Acredita-se que as análises apresentadas auxiliam em um melhor entendimento da realidade do sistema e servem como substrato para proporcionar uma melhor gestão e alocação de recursos destinados ao atendimento desse público. Como trabalho futuro, pretende-se estender a aplicação dos métodos aqui investigados a outros casos, bem como desenvolver um arcabouço que permita a avaliação integrada das trajetórias de pacientes sob diferentes perspectivas. O que possibilita serem feitas indicações sobre potenciais trajetórias que otimizam o sistema como um todo.

\section{Agradecimentos}

À CAPES, FAPERJ, FAPEMIG, FAPESP e ao CNPq, pelo apoio financeiro.

\section{Referências}

Bailey, T. C. (2001). Spatial statistical methods in health. Cadernos de Saúde Pública, 17(5):1083-1098.

Baker, K., Dunwoodie, E., Jones, R. G., Newsham, A., Johnson, O., Price, C. P., Wolstenholme, J., Leal, J., McGinley, P., Twelves, C., and Hall, G. (2017). Process mining 
routinely collected electronic health records to define real-life clinical pathways during chemotherapy. International Journal of Medical Informatics, 103:32-41.

Espinoza, C., Pascual, J., Ramis, F., Bórquez, D., and Sepúlveda, J. A. (2014). Real-time simulation as a way to improve daily operations in an emergency room. In Proceedings of the Winter Simulation Conference 2014, pages 1445-1456. IEEE.

Ito, M., Appel, A. P., de Santana, V. F., and Moyano, L. G. (2017). Analysis of the existence of patient care team using social network methods in physician communities from healthcare insurance companies. Studies in Health Technology and Informatics.

Kempa-Liehr, A. W., Lin, C. Y. C., Britten, R., Armstrong, D., Wallace, J., Mordaunt, D., and O'Sullivan, M. (2020). Healthcare pathway discovery and probabilistic machine learning. International Journal of Medical Informatics, 137.

Lismont, J., Janssens, A. S., Odnoletkova, I., vanden Broucke, S., Caron, F., and Vanthienen, J. (2016). A guide for the application of analytics on healthcare processes: A dynamic view on patient pathways. Computers in Biology and Medicine, 77:125-134.

Ministério da Saúde (2000). Programa de humanização no pré-natal e nascimento. Disponível em: http://bvsms.saude.gov.br/bvs/saudelegis/gm/2000/prt0570_01_06_2000_r ep.html.

Ministério da Saúde (2019). Sistema Único de Saúde (SUS). Disponível em: http://ww w.saude.gov.br/sistema-unico-de-saude\#principios.

Pollack, C. E., Weissman, G. E., Lemke, K. W., Hussey, P. S., and Weiner, J. P. (2013). Patient sharing among physicians and costs of care: a network analytic approach to care coordination using claims data. Journal of general internal medicine, 28(3):459465.

Saldanha, R. d. F., Xavier, D. R., Carnavalli, K. d. M., Lerner, K., and Barcellos, C. (2019). Estudo de análise de rede do fluxo de pacientes de câncer de mama no brasil entre 2014 e 2016. Cadernos de Saúde Pública, 35:e00090918.

Souza, F., Silva, L., and Roveri, E. (2008). Desenvolvimento de um sistema para o gerenciamento das internações e fluxo de pacientes entre hospitais e cidades de uma região. In Anais do XI Congresso Brasileiro de Informática em Saúde. Campos do Jordão: Universidade Federal de Minas Gerais, pages 1-6.

São Paulo (2010). Atenção à gestante e à puérpera no SUS-SP: Manual Técnico do Pré-natal e Puerpério. São Paulo. 234p.

São Paulo (2020). Coordenadoria de Regiões de Saúde - CRS. Disponível em: http: //www.saude.sp.gov.br/coordenadoria-de-recursos-humanos/orgaos-sub-setoriais/coo rdenadoria-de-regioes-de-saude-crs.

Thorwarth, M. and Arisha, A. (2012). A simulation-based decision support system to model complex demand driven healthcare facilities. In Proceedings of the 2012 Winter Simulation Conference (WSC), pages 1-12. IEEE. 